\title{
ASSESSMENT OF A POINT-OF-CARE DEVICE FOR MEASURING CREATININE IN A COMMUNITY SCREENING PROGRAM FOR CHRONIC KIDNEY DISEASE
}

\author{
Brooke Ann Spaeth, Anne K Shephard, Mark DS Shephard, Timothy H Mathew
}

\begin{abstract}
Background: Chronic kidney disease (CKD) is a major contemporary global health problem. Creatinine measurement for the calculation of estimated glomerular filtration rate is an important component of assessing CKD risk. A point-of-care test for creatinine using capillary sampling is required as part of a screening assessment.
\end{abstract}

Objectives: Evaluate the analytical performance of a modified point-of-care testing method for whole blood creatinine (Nova Biomedical StatSensor whole blood creatinine analyser) relative to a laboratory method.

Design and methods: Conduct a patient comparison study between the point-of-care testing and laboratory methods in a rural community setting. Calculate measures of imprecision and assess the ability of the POCT method to determine staging of CKD compared to the laboratory.

Results: Between-device imprecision averaged 8.8\%. The StatSensor devices showed a positive bias of approximately $14 \%$ for whole blood creatinine measurement compared to the laboratory method, leading to more than $40 \%$ of community patients being staged differently for CKD risk with approximately $25 \%$ more abnormal results.

Conclusions: The StatSensor whole blood creatinine point-of-care device remains analytically unsound for use as a screening device for CKD.

Key Words: chronic kidney disease; point-of-care testing; creatinine; estimated glomerular filtration rate. 


\section{BACKGROUND}

The burden of chronic kidney disease (CKD) continues to increase both in Australia and throughout the world [16]. A recent article highlighted the evolving importance of chronic kidney disease - "from subspecialty to global health problem" [1]. CKD is often described as a 'silent' disease due to its asymptomatic nature. In Australia, approximately $10 \%$ of adults presenting to their family practice have CKD and $80 \%$ have at least one risk factor for CKD [7]. Kidney Health Australia, in partnership with Flinders University International Centre for Point-of-Care Testing, has undertaken targeted screening in primary care settings for members of the general population considered at high risk for CKD through an initiative called KEY (Kidney Evaluation for You) [8]. The screening strategy incorporates the use of point -of-care testing for markers of risk including blood creatinine, urine albumin:creatinine ratio (ACR), and blood pressure [9]. Creatinine measurement provides an estimate of the glomerular filtration rate (eGFR), which can be used to stage CKD [10-12]. The Abbott i-STAT device (Abbott Point of Care, Princeton, NJ, USA) has been the device of choice for point-of-care creatinine measurements in KEY due to its analytical reliability [5]. However the i-STAT requires $60 \mu \mathrm{L}$ of venous whole blood to measure creatinine. For patient convenience, a POC device that uses capillary (fingerprick) whole blood would provide a more practical option for primary-care based CKD screening. In 2010, we evaluated the Nova StatSensor (Nova Biomedical, Waltham, MA, USA) hand- held POC device for creatinine, which used $1.2 \mu \mathrm{L}$ of capillary whole blood and provided a creatinine (and associated eGFR result) in 30 seconds. However, its analytical performance did not meet specifications for both precision and accuracy [13]. The manufacturer of the device subsequently made amendments to the device designed to improve its analytical performance characteristics. We re -evaluated the modified device in a rural community setting as part of the KEY program and the findings of this study are reported here.

\subsection{DESIGN AND METHODS \\ 2.1 Setting}

The device evaluation was conducted as part of a community screening event held by Kidney Health Australia for citizens over the age of 50 years from the rural farming town of Kyabram, 200 kilometres north of Melbourne, Victoria, Australia. The surrounding community has a population of approximately 12,000 people, of which $2 \%$ are of Aboriginal descent [14].

\subsection{Patient Samples}

Venous and capillary whole blood samples were collected from 111 community participants (35 male, 76 female, mean age 68 years). After consent was obtained, a venous whole blood specimen was collected into a lithium heparin anticoagulant tube and sent to the nearest laboratory (Monash, Melbourne, Victoria) for analysis of plasma creatinine. A capillary specimen was then obtained and immediately analysed on two StatSensor creatinine analysers (Nova Biomedical, USA) using the same reagent strip lot number.

\subsection{Test method}

To perform the point-of- care test for creatinine, the reagent strip was 
inserted into the analyser prior to sample application. Calibration information is encoded within this strip. Whole capillary blood was then applied to the reagent strip and the creatinine result was displayed in 30 seconds. Finger-prick analyses were conducted according to manufacturer instructions by a trained medical scientist (BS). Quality Control testing was performed at the beginning and at the end of each day of screening using the manufacturer's recommended quality control material.

\subsection{Comparative laboratory method}

Venous whole blood samples were sent to the laboratory and measured on a Beckman UniCel ${ }^{\circledR}$ DxC800 Synchron (Beckman Coulter, Australia) using the Jaffe rate method to determine the concentration of creatinine. This method is traceable to the isotope dilution mass spectrometry (IDMS) reference method.

\subsection{Accuracy}

The point -of-care creatinine result on each StatSensor analyser was compared to the creatinine result from the laboratory method using Passing Bablok linear regression analysis. Differences between results were plotted against the laboratory method using Bland Altman difference analysis. Estimated GFR on the StatSensor (using CKD-EPI equation) was also plotted against eGFR from the laboratory method [15]. The staging of CKD using the StatSensor eGFR was then compared to the laboratory staging of CKD.

\subsection{Statistical Analyses}

Statistical analyses were performed using the statistical package Analyse-it for Microsoft Excel (clinical laboratory version 2.21).

\subsection{RESULTS \\ 3.1 Imprecision}

Between device imprecision (calculated from matched patient results from each StatSensor device) was $8.8 \%$ (capillary blood, creatinine range 50 to 212 $\mu \mathrm{mol} / \mathrm{L}, \mathrm{n}=109$ ).

\subsection{Method comparison}

Creatinine concentrations in samples tested by the laboratory ranged from 48 to $168 \mu \mathrm{mol} / \mathrm{L}$ (mean $83 \mu \mathrm{mol} / \mathrm{L}$ ). Table 1 summarises the method correlation statistics for StatSensor devices 1 and 2 versus the laboratory (Jaffe) method.

Table 1 - Method correlation statistics for the StatSensor versus the laboratory method.

\begin{tabular}{ccccccccc}
\hline Device & $\begin{array}{c}\mathrm{PB}^{\mathrm{a}} \\
\text { Slope }\end{array}$ & $\begin{array}{c}\mathrm{PB}^{\mathrm{a}} \\
\text { Intercept }\end{array}$ & $\mathrm{r}^{\mathrm{b}}$ & $\begin{array}{c}\text { Mean } \\
\text { Laboratory } \\
\text { Creatinine } \\
(\mu \mathrm{mol} / \mathrm{L})\end{array}$ & $\begin{array}{c}\text { Mean } \\
\text { StatSensor } \\
\text { Creatinine } \\
(\mu \mathrm{mol} / \mathrm{L})\end{array}$ & $\begin{array}{c}\text { Mean Bias } \\
(\mu \mathrm{mol} / \mathrm{L})\end{array}$ & $\begin{array}{c}95 \% \text { limits } \\
\text { of Agreement } \\
(\mu \mathrm{mol} / \mathrm{L})\end{array}$ & $\mathrm{n}$ \\
\hline StatSensor 1 & 1.21 & 0.71 & 0.81 & 83 & 99 & 16.4 & -12.1 to 44.8 & 109 \\
StatSensor 2 & 1.18 & -4.02 & 0.84 & & 93 & 10.1 & -16.2 to 36.4 & 109
\end{tabular}

${ }^{\mathrm{a}} \mathrm{PB}=$ Passing Bablock linear regression.

${ }^{b} \mathrm{r}=$ correlation coefficient. 
Both StatSensor analysers produced higher results than the laboratory method (average bias 13.7\%) and there was a statistically significant difference between creatinine results on the two analysers (paired t test $\mathrm{p}<0.001$ ).

Due to the over estimation of creatinine values with the StatSensor (and therefore an underestimation of eGFR values [EKD-EPI]), $48 \%$ of patients were staged differently for CKD with StatSensor 1 and $41 \%$ with StatSensor 2 (see Fig. 1) compared to the staging of CKD from laboratory results. There were $31 \%$ more (34 participants) abnormal results (eGFR <60) with StatSensor 1 and $21 \%$ (23 participants) more abnormal results with StatSensor 2 compared to laboratory results. One patient $(0.9 \%)$ with StatSensor 1 and three patients $(2.8 \%)$ with StatSensor 2 had normal eGFR results $(>60)$ from StatSensor testing but abnormal eGFR on laboratory testing (see Fig. 1).

Fig 1: Plot of eGFR (mL/min) for Nova StatSensor 1 and 2 versus laboratory method.
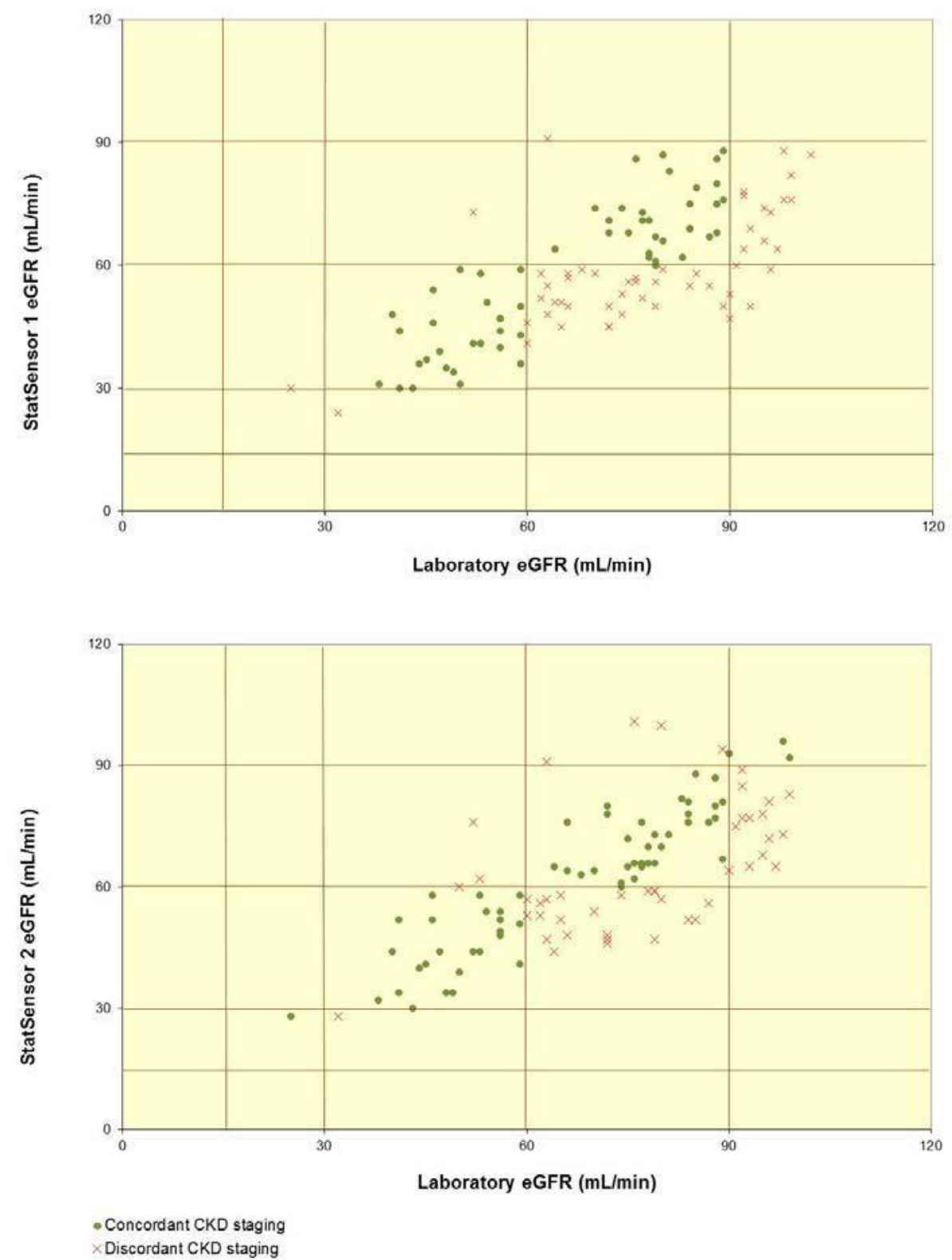


\subsection{DISCUSSION}

With the global prevalence of CKD continuing to escalate, the need for simple, minimally invasive screening tests for identifying CKD risk is becoming increasingly important. An analytically sound whole blood creatinine measurement that can be performed using finger-prick sampling would be most useful in this context. The StatSensor creatinine analyser fits many of the criteria for a screening device, given it is handheld, requires a small sample volume, is battery operated and has the onboard capacity to automatically convert creatinine concentration to an eGFR value. However, in a previous evaluation of this device conducted in a hospital environment, we found its analytical performance was poor. The results of the current study, conducted in a rural primary care setting, indicate that the modified StatSensor devices still do not meet analytical specifications. Between-device imprecision had not improved $7.8 \%$ in the original evaluation and $8.8 \%$ in the present study) and creatinine results varied significantly between the two analysers. Based on this current study the StatSensor device is unsuitable for use as a screening tool in routine practice.

\subsection{CONCLUSIONS}

Despite the efforts of the company to improve the performance of the device, the findings of this study indicate the StatSensor whole blood creatinine device remains analytically unsound for use as a screening device for CKD.

SOURCES OF SUPPORT THAT REQUIRE ACKNOWLEDGEMENT: devices, reagents and quality control materials to support the point-of -care testing conducted in this project and provided financial support for laboratory analysis of venous samples.

Nova Biomedical provided 


\section{REFERENCES}

[1] Eckhardt K-U, Coresh J, Devuyst O, Johnson R, Kottgen A, Levey A et al. (2013). Evolving importance of kidney disease: from subspecialty to global health burden. Lancet, 382: 158-169.

[2] Jha V, Garcia-Garcia G, Iseli K, Li Z, Naiker S, Plattner B et al. (2013). Chronic kidney disease; global dimension and perspectives. Lancet, 382: 260-272.

[3] Jha V, Wang A-M, Wang H. (2012). The impact of CKD identification in larger countries: the burden of illness. Nephrol Dialysis Transplant, 27: 32-38.

[4] Levey A, Coresh J. (2012). Chronic kidney disease. Lancet, 379: 165-180.

[5] Ayodele O, Alebiousi C. (2010). Burden of chronic kidney disease; an international perspective. Adv Chronic Kid Dis, 17: 215-224.

[6] Couser W, Remuzzi G, Mendis S, Tonelli M. (2011). The contribution of chronic disease to the global burden of major non-communicable diseases. Kidney Int, 80: 1258-1270.

[7] Chronic kidney disease (CKD) management in general practice $\left(2^{\text {nd }}\right.$ edition). Kidney Health Australia, Melbourne 2012. Available at www.kidney.org.au [last accessed 1 September 2014].

[8] Mathew T, Corso O, Ludlow M, Boyle A, Cass A, Chadban S, et al. (2010). Screening for chronic kidney disease in Australia: a pilot study in the community and workplace. Kidney Int, 77 (Supp 116): S9-S16.

[9] Shephard A, Shephard M, Halls H, Corso O, Mathew T. (2011). Innovative use of point-of-care testing for chronic kidney disease screening. Point of Care, 10: 98-101.
[10] Polkinghorne K. (2014). Estimated glomerular filtration rate versus albuminuria in the assessment of kidney function: What's more important? Clin Biochem Revs, 35: 67-73.

[11] Shephard M. (2011). Point-of-care testing and creatinine measurement. Clin Biochem Revs, 32: 109-114.

[12] Peake M, Whiting M. (2006). Measurement of serum creatinine - current status and future goals. Clin Biochem Revs, 27:173-84.

[13] Shephard MD, Peake MJ, Corso O, Shephard AK, Mazzachi BC, Spaeth BA et al. (2010). Assessment of the NovaStatSensor whole blood point-of-care creatinine analyser for the measurement of kidney function in screening for chronic kidney disease. Clin Chem Lab Med, 48: 1113-1119.

[14] Australian Bureau of Statistics (2011). Information Paper: Census of Population and Housing -- Products and Services, 2011 (cat. no. 2011.0.55.001). Available at www.abs.gov.au [last accessed 8 September 2014].

[15] Matsushita K, Mahmoodi BK, Woodward M, Emberson JR, Jafar TH, Jee SH et al. (2012). Comparison of risk prediction using the CKD-EPI equation and the MDRD study equation for estimated glomerular filtration rate. JAMA, 37 (18) 1941-1951. 